\title{
Velopharyngeal Dysfunction
}

\author{
Albert S. Woo, MD ${ }^{1}$ \\ ${ }^{1}$ Cleft Palate-Craniofacial Institute and Department of Surgery, \\ Washington University in St. Louis, St. Louis, Missouri \\ Semin Plast Surg 2012;26:170-177.
}

\begin{abstract}
Address for correspondence and reprint requests Albert S. Woo, MD, Director, Cleft Palate-Craniofacial Institute, Assistant Professor, Plastic Surgery, Department of Surgery, Washington University in St. Louis, 660 South Euclid Avenue, Campus Box 8238, St. Louis, MO 63110 (e-mail: WooA@wustl.edu).
\end{abstract}

\begin{abstract}
Velopharyngeal dysfunction (VPD) is a generic term which describes a set of disorders resulting in the leakage of air into the nasal passages during speech production. As a result, speech samples can demonstrate hypernasality, nasal emissions, and poor intelligibility. The finding of VPD can be secondary to several causes: anatomic, musculoneuronal, or behavioral/mislearning. To identify the etiology of VPD, patients must undergo a thorough velopharyngeal assessment comprised of perceptual speech evaluation and functional imaging, including video nasendoscopy and speech video-

Keywords

- velopharyngeal dysfunction

- insufficiency

- cleft palate

- hypernasal speech fluoroscopy. These studies are then evaluated by a multidisciplinary team of specialists, who can decide on an optimal course for patient management. A treatment plan is developed and may include speech therapy, use of a prosthetic device, and/or surgical intervention. Different surgical options are discussed, including posterior pharyngeal flap, sphincter pharyngoplasty, Furlow palatoplasty, palatal re-repair, and posterior pharyngeal wall augmentation.
\end{abstract}

Velopharyngeal dysfunction (VPD) refers to any situation in which an individual is unable to completely close the nasal airway during speech. The velopharyngeal mechanism is comprised of a complex group of structures that act in unison to control airflow through the nose and mouth by elevation of the soft palate and constriction of both the lateral and posterior pharyngeal walls (-Fig. 1). Any disruption in this mechanism may result in abnormal, poorly intelligible speech. VPD can manifest as hypernasality, nasal emission, decreased vocal intensity, and/or facial grimacing. ${ }^{1}$ Moreover, patients who suffer from VPD will frequently develop maladaptive articulations to compensate for their speech difficulties. ${ }^{2}$

Numerous etiologies can be responsible for this failure of normal speech production. Myoneurogenic problems can impair muscle control or affect muscle programming. Anatomic irregularities can present as a tissue deficit, structural problems that affect function, or even mechanical interference preventing normal closure. Mislearning comprises a host of etiologies whereby the patient has developed abnormal usage of the velopharyngeal mechanism despite the absence of other pathology. ${ }^{3}$

Velopharyngeal dysfunction is a carefully chosen term that simply denotes the presence of incomplete velopharyngeal closure without making suggestions as to its cause. Use of this nomenclature has gained increasing favor by experts, replacing the previous designation, velopharyngeal insufficiency (VPI). This helps to avoid confusion, as VPI has been differentially interpreted as denoting insufficiency, incompetence, and inadequacy-terms that may be similar, but are not synonymous and potentially implicate the cause of the dysfunction rather than describe the clinical finding. VPD is seen in roughly 20 to $30 \%$ of individuals who have undergone cleft palate repair, ${ }^{4,5}$ and 5 to $10 \%$ of patients with a submucous cleft palate (SMCP). ${ }^{6}$

\section{Velopharyngeal Assessment}

The assessment of velopharyngeal function is best performed in the setting of a multispecialty team evaluation composed of a speech-language pathologist (SLP), otolaryngologist, prosthodontist, and plastic surgeon. Multiple modalities should be utilized to perform a complete evaluation of the patient. After a thorough review of the patient's history, the standard workup involves perceptual speech evaluation, followed by video nasoendoscopy (VNE) and multiview speech videofluoroscopy (SVF). ${ }^{7,8}$

There is considerable variation in the utilization of imaging studies to guide treatment of VPD. Different institutions
Issue Theme Pediatric Plastic Surgery-Clefts; Guest Editor, Edward

P. Buchanan, MD
Copyright (c) 2012 by Thieme Medical Publishers, Inc., 333 Seventh Avenue, New York, NY 10001, USA. Tel: +1(212) 584-4662.
DOI http://dx.doi.org/ 10.1055/s-0033-1333882. ISSN 1535-2188. 


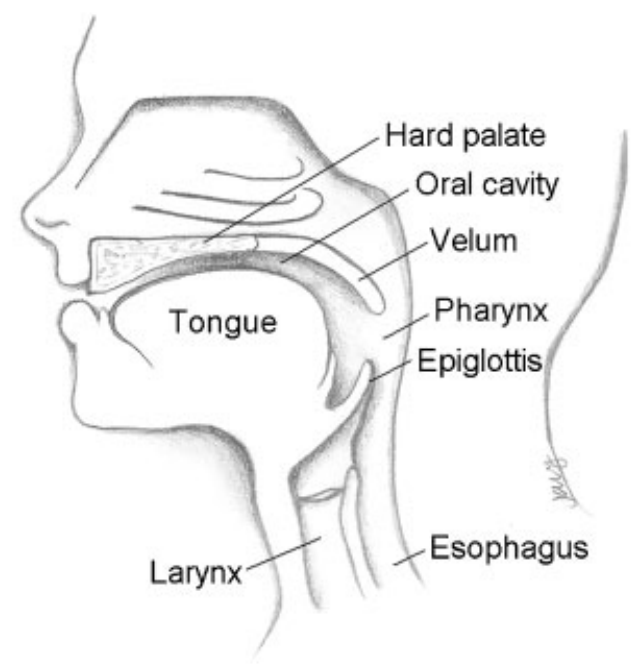

Fig. 1 Velopharyngeal anatomy in the sagittal plane.

will preferentially utilize VNE or SVF or other novel imaging modalities; other institutions use both studies for comprehensive evaluation. Lipira et $\mathrm{al}^{9}$ evaluated the relative benefits of videofluoroscopy versus nasoendoscopy and concluded that both studies were best used in tandem to optimally evaluate patients with VPD.

\section{Perceptual Speech Evaluation}

The initial diagnosis of VPD is made on perceptual speech evaluation (PSE) conducted by a specialized SLP. During this examination, the SLP will perform multiple tests to elicit the etiology of the VPD and determine whether further diagnostic imaging would be beneficial. The diagnosis of velopharyngeal dysfunction (VPD) encompasses a range of stigmatizing speech impairments characterized by inappropriate nasal resonance, nasal air emission, nasal turbulence, grimacing, and nasalized plosives. ${ }^{1,2}$

Abnormal closure of the nasal valve can result in abnormal resonance, which is a descriptor of where sound moves throughout the vocal tract. Hypernasality is a resonance disorder characterized by abnormal sound escape into the nasal cavity during speech, particularly with the use of vowels. Hyponasality, on the other hand, describes a situation where there is decreased resonance in the nasal cavity, which can develop secondary to blockage of the upper airway during upper respiratory infection or other means of obstruction (i.e., overly large pharyngeal flap).

Nasal emission describes the escape of air into the nose during speech. This is especially notable during the production of pressurized consonants, such as $\mathrm{s} / \mathrm{z}$ and $\mathrm{p} / \mathrm{b}$. Emissions may be easily detected by placement of a mirror underneath the nostril during the speech sample. Nasal turbulence (or nasal rustle) is a phenomenon that occurs when air is leaking through a small residual velopharyngeal opening. The resulting sound can be distracting and is most notable with the production of oral pressure consonants.

Inadequate intraoral air pressure is a common finding in patients with VPD, who can lose pressure during the produc- tion of oral speech sounds secondary to leakage of air into the nasal cavity. Several compensatory mechanisms can develop to make up for the loss of pressure. One such mechanism is nasal grimace, which is an abnormal constriction of the nostrils during speech production. This phenomenon occurs as a subconscious attempt to block airflow through the nose when nasal emissions occur.

Compensatory or maladaptive misarticulations describe a host of speech production disorders that may have spontaneously developed to compensate for reduced intraoral air pressure. One must always remember, however, that articulation disorders may result from a myriad of differing etiologies unassociated with VPD; it is the job of an SLP experienced in cleft speech abnormalities to parse out articulation disorders from those resulting from a structural abnormality.

Based on the perceptual speech examination, the SLP can establish the presence of VPD and develop suspicions as to its underlying cause. Nevertheless, the specific etiology of the VPD and the degree of nasopharyngeal valve dysfunction can only be determined with anatomic visualization. This is achieved via the modalities of video nasoendoscopy and speech videofluoroscopy.

\section{Video Nasopharyngeal Endoscopy}

Video nasopharyngeal endoscopy (VNE) is a technique that allows direct visualization of the velopharyngeal mechanism during speech production. In this procedure, an endoscopist inserts a small, flexible nasopharyngoscope into an anesthetized nostril. The scope is passed through the middle meatus of the nose and rests in the posterior nasal passages. Optimal viewing of the soft palate, lateral pharyngeal walls, and posterior pharynx allows the endoscopist to establish an overall assessment of velopharyngeal function.

Once appropriate positioning and visualization has been obtained, an SLP guides the patient through repetition of a standardized speech sample tailored to the patient's abilities. Each study (composed of both video and audio data) is recorded for later review by the multispecialty group.

VNE evaluation allows the direct visualization of the degree of maximal velopharyngeal closure, the position and function of the levator musculature, length and quality of the soft palate, and the degree of motion of the lateral pharyngeal walls and the posterior pharynx. Moreover, VNE is the best study to establish assessment of an overall closure pattern based on the directional movements of different components of the velopharynx (-Fig. 2). An understanding of the pattern of closure and the degree of movement of different musculature will play a critical role in the decisionmaking process for treatment.

One limitation of this study is the inability to quantitatively measure pertinent anatomic findings, such as gap size. Estimates, however, can be made based upon standardized reporting techniques. ${ }^{10}$ Younger patients may also have difficulty with cooperating with the speech sample during the examination, as nasopharyngoscopy may be an awkward and uncomfortable procedure even at the hands of an experienced endoscopist. 
A
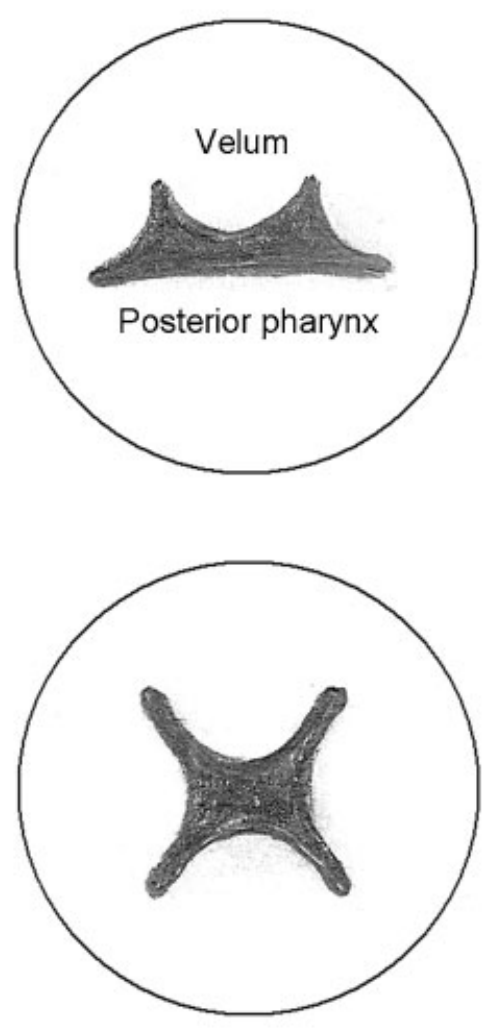

C

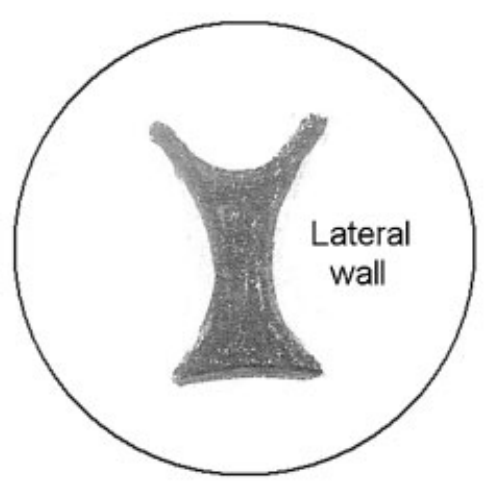

B

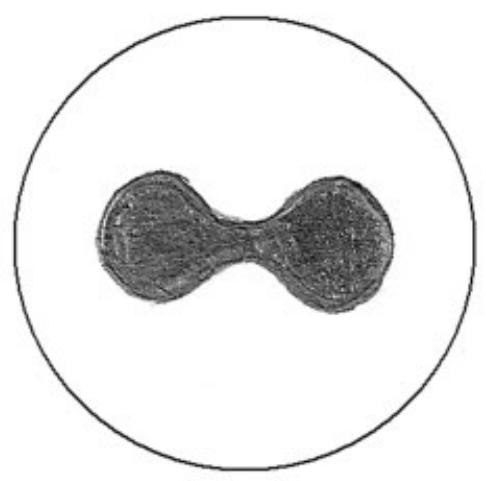

Fig. 2 Velopharyngeal closure patterns are demonstrated. Note that the velum is anterior and the posterior pharyngeal wall is inferior. (A) Coronal: There is significant movement of the velum with less movement of the lateral pharyngeal walls. (B) Sagittal: The lateral pharyngeal walls have excellent motion and provides the predominant source of closure. The velum demonstrates less movement. (C) Circular: Good movement is seen from the velum and lateral walls, resulting in a circular pattern of closure. A Passavant ridge may also contribute to this phenomenon. (D) Bowtie: Closure is primarily due to the velum and possibly a Passavant ridge from the posterior pharynx. Lateral wall movement is poor.

\section{Speech Videofluoroscopy}

Multiview speech videofluoroscopy is another modality that provides visualization of the velopharyngeal apparatus during speech production. This procedure is performed as a collaborative effort between a radiologist and an SLP. Highdensity contrast material is syringe-injected via both nares prior to examination. An SLP then guides the patient through the repetition of a standardized speech sample personalized to the patient's abilities. This procedure is typically performed in both the lateral and anteroposterior (AP) views and is recorded for subsequent review.

Radiographic studies tend to be better tolerated than VNE, especially among younger patients, and are able to provide some quantitative data regarding velopharyngeal closure. However, patterns of closure are more difficult to assess. SVF also necessitates some exposure to radiation and is limited by the individual's ability to cooperate.

\section{Classification of Velopharyngeal Dysfunction}

The management of VPD differs significantly depending on its etiology, which is a critical factor in decision making and can be classified into several categories. Anatomic causes are most common and are typically associated with a previously repaired cleft palate. Often referred to as velopharyngeal insufficiency, the soft palate may be too short (or "insuffi- cient") to permit adequate approximation of the velum to the posterior pharynx. The palate may also contain a significant amount of scar tissue, which can shorten the palate and decrease the mobility of the velum. Further, aberrant insertion of the levator veli palatini muscles can inhibit optimal palatal movement. Fistulas anywhere within the palate can lead to abnormal intraoral air escape, and tonsillar hypertrophy or scarring of the posterior tonsillar pillars can also serve as a barrier to normal closure of the velum against the posterior pharyngeal wall.

Neuromuscular etiologies can also result in VPD and are occasionally referred to as velopharyngeal incompetence. Childhood apraxia of speech is a motor speech disorder that hinders appropriate coordination of muscle movements for appropriate function. Hypernasality increases with connected speech and is associated with inconsistent articulation errors. Neurologic impairment, congenital abnormalities, or traumatic/iatrogenic injury are among some of the other myoneuronal etiologies that can render the velopharyngeal apparatus "incompetent," leading to VPD.

Articulation disorders due to mislearning are a frequent source of VPD. Behavioral (rather than structural) etiologies typically present with consistent phoneme-specific nasal emissions or hypernasality rather than the pervasive nonspecific hypernasality present when velopharyngeal closure is incomplete. Nevertheless, almost all patients with anatomic 


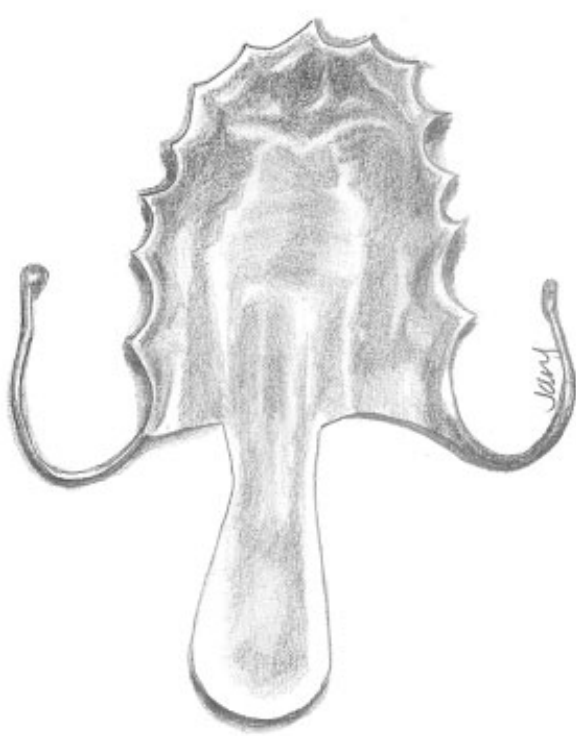

Fig. 3 Diagram of a palatal lift, which is stabilized on the dentition and is designed to elevate the soft palate tissues with its posterior extension.

causes of VPD present with compensatory misarticulations to optimize speech production. Differentiating between the two types of misarticulations (mislearning vs compensatory) can be a challenging task for the SLP. Regardless of etiology, most children with VPD will benefit from an appropriate course of speech therapy to optimize their ability to communicate.

\section{Nonsurgical Treatment Options}

Prosthetic options exist to aid in the treatment of VPD and may be utilized temporarily or serve as a permanent solution for nonsurgical candidates. Prostheses typically are available in the form of a palatal lift or an obdurator. Each device is custom-made for the individual by a maxillofacial prosthodontist and is designed to anchor into the maxillary dentition, similar to a retainer. Palatal lifts contain posterior extensions that press upward along the soft palate, physically displacing it superiorly in an attempt to aid velopharyngeal closure (-Fig. 3). These devices are best utilized in situations of velopharyngeal incompetence, where the palate suffers from hypomobility, poor muscle coordination or paralysis, but has adequate soft tissue length.

Soft palate obdurators or speech aid prostheses are more effective in velopharyngeal insufficiency, where the palate has inadequate tissue length. These devices are similar in appearance to obdurators, but are designed with additional material that extends beyond the soft tissues to aid in achieving velopharyngeal closure.

\section{Surgical Treatment Options}

Patients with a history of previously repaired cleft palate and anatomic findings of VPD are frequently candidates for surgical intervention. Once the decision for surgery has been established, a choice must be made as to which intervention would best fit the needs of the patient. The two most commonly discussed procedures for correction of VPD remain the posterior pharyngeal flap and the sphincter pharyngoplasty. Both procedures work to decrease the size of the residual velopharyngeal port.

More recently, procedures designed to improve palatal closure have gained increasing popularity. The Furlow palatoplasty and palatal re-repair are two techniques performed to either lengthen the palate or otherwise tighten the levator sling. Some authors have also reported a modicum of success with posterior pharyngeal wall augmentation procedures.

Due to the plethora of surgical and nonsurgical options, a multidisciplinary team consisting of a plastic surgeon, speech therapist, otolaryngologist, and maxillofacial prosthodontist is thought to be best equipped for optimal decision making. Surgical procedures can be tailored to the patient's specific anatomy, as visualized on VNE and SVF studies. Based upon the imaging, a pattern of closure can be determined as well as the size of the defect.

Velopharyngeal closure patterns can be classified as coronal, sagittal, circular, or bowtie (-Fig. 2). Surgical management should differ based upon the type of deformity present. Pharyngeal flaps are designed to bring tissue into the central portion of the velopharynx. Therefore, they are best utilized to correct central gaps (sagittal or circular patterns of closure) where good lateral pharyngeal wall motion is visualized on VNE or SVF in the AP dimension. ${ }^{11}$ Sphincter pharyngoplasty, on the other hand, brings in tissue laterally toward the center and appears most useful for lateral defects (coronal and bowtie patterns), especially when lateral wall motion is poor. Furlow palatoplasty has shown success primarily in smaller central gaps, especially in circumstances where evidence exists of diastasis of the levator muscle sling (i.e., midline notch on VNE). Posterior pharyngeal augmentation procedures are similarly utilized for very small residual defects. Little consensus exists in regards to the treatment of large "black hole" deformities, which tend to have the poorest results when reconstruction is attempted. Some have noted success with sphincter pharyngoplasty alone ${ }^{12}$ or with wide, nearly obstructing pharyngeal flaps. Others have suggested that results are best when palatal lengthening procedures such as Furlow palatoplasty are performed in conjunction with a sphincter pharyngoplasty. ${ }^{13}$

Despite the theories and preferences for reconstruction that have been noted above, little evidence exists suggesting whether pharyngeal flap or sphincter pharyngoplasty is superior to the other. Rather, both procedures appear to have equivalent efficacy when performed by experienced surgeons. ${ }^{14}$ In a prospective, randomized trial, the VPI Surgical Trial Group ${ }^{15}$ evaluated 97 patients at five international centers who presented with VPD. Individuals were randomized to either of the procedures, which were performed in a standardized fashion by each of the surgeons involved. At 3 months following surgery, pharyngeal flap patients were twice as likely to demonstrate resolution of hypernasality. However, at 12 months, there was no statistically significant difference in outcomes. 


\section{Pharyngeal Flap}

The primary concept behind the pharyngeal flap is the creation of a static wall of mucosa connecting the soft palate to the posterior pharynx, thereby decreasing airflow through the velopharyngeal port. The nasal airway is preserved through two lateral openings on either side of the flap. The success of the operation depends on adequate mobility of the lateral pharyngeal walls, which should constrict inward during speech production to limit airflow through the nose when producing pressure consonants.

The first pharyngeal flap procedure was introduced by Schoenborn ${ }^{16}$ in 1875 . Originally inferiorly based, he had converted his technique to a superiorly-based procedure after performing 20 operations by $1886 .{ }^{17}$ This procedure was brought to the United States by Padgett, ${ }^{18}$ who used a superiorly-based flap for correction of dehisced cleft palate repairs. Variations of the procedure became widely adopted in the 1950s. In 1973, the modern pharyngeal flap was introduced by Hogan, ${ }^{19}$ who popularized the idea of lateral port control and discussed coverage of the raw surface of the flap to prevent postoperative contracture. This concept was taken a step further by Shprintzen, ${ }^{20}$ who described the creation of flaps that were tailored based on lateral pharyngeal wall excursion. It is now standard dictum that lateral wall motion is critical for effective closure of the lateral pharyngeal ports following pharyngeal flap surgery. ${ }^{11}$ Hence, this procedure is thought to be most effective for sagittal or circular closure patterns, with adequate lateral wall motion.

The standard technique for elevation of a superiorly-based pharyngeal flap (-Fig. 4) involves division of the soft palate in the midline to aid in visualization of the posterior pharynx. Longitudinal incisions are made in the posterior pharyngeal wall converging into a point along the inferior border. The flap is then elevated at the prevertebral fascia to the level of the first cervical vertebrae. The nasal lining on either side of the soft palate is then released to serve as lining for the undersurface of the pharyngeal flap. The pharyngeal flap is inset into the base of the incised soft palate. Lateral port size is often controlled by placement of red rubber catheters (10-12 French) on either side to maintain adequate airflow outlets. Control of port size is important because an overly obstructing flap will result in hyponasality with excessive mouth breathing and even obstructive sleep apnea; in contrast, a flap that is too narrow will not adequately correct the VPD. The nasal lining is then sutured to the raw surface of the pharyngeal flap. The soft palate is closed at the midline as is the donor site along the posterior pharynx.

\section{Sphincter Pharyngoplasty}

The sphincter pharyngoplasty technique serves conceptually as a "speed bump" or extension of the lateral and posterior pharyngeal walls, which helps to close up the size of the velopharyngeal gap, making it easier for the soft palate to achieve closure during dynamic movement. Although the nasal airway remains centrally, it is significantly decreased in size. The success of this procedure hinges upon adequate function of the levator veli palatini muscles, which serve to close the central port during speech production. Lateral wall motion is less important, as the flap brings in tissue on either side.

The procedure was first introduced by Hynes in 1950,21 who originally described elevation of the salpingopharyngeus muscles and mobilization into a transverse orientation for augmentation of the posterior pharynx. Eventually, he

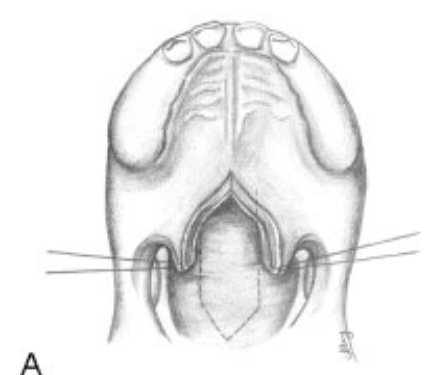

A

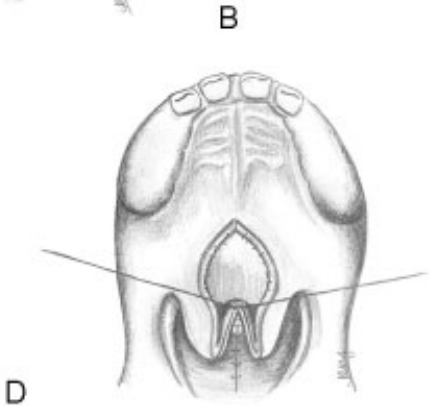

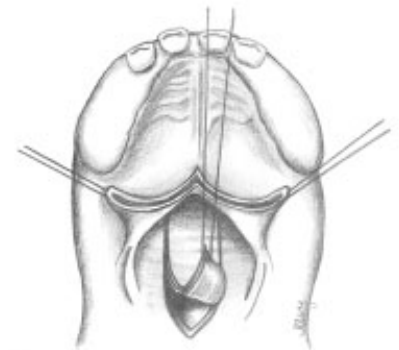

C

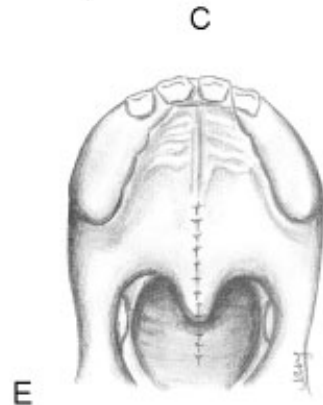

Fig. 4 Technique for pharyngeal flap surgery. (A) The soft palate is divided at the midline and retracted laterally. A superiorly-based flap is then designed along the posterior pharynx (dotted lines). (B) The posterior pharyngeal flap is elevated from inferior-to-superior at the level of the prevertebral fascia. (C) The flap is inset into the nasal mucosa of the soft palate. Laterally, nasal mucosa flaps from the soft palate are elevated to serve as lining for the raw edge of the pharyngeal flap. (D) The nasal mucosa flaps are inset onto the undersurface of the pharyngeal flap. The donor site of the pharyngeal flap has also been closed primarily. (E) The oral mucosa is closed. Note that the pharyngeal flap is not visible after closure is completed. 
A

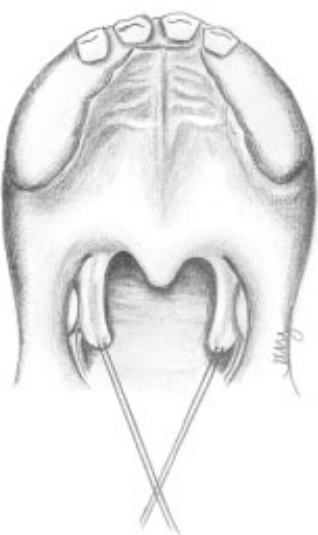

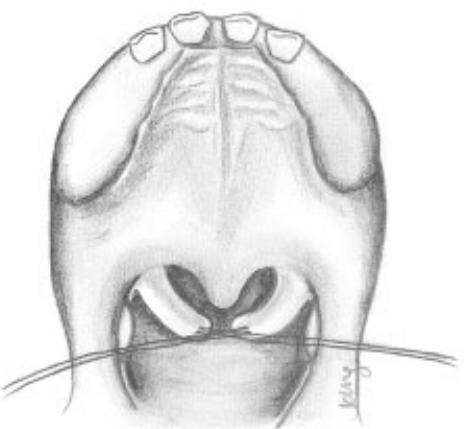

B

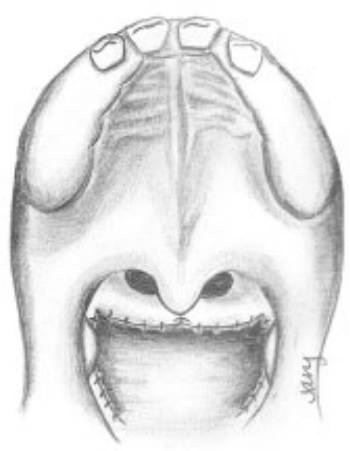

C

Fig. 5 Technique for sphincter pharyngoplasty. (A) Musculomucosal flaps are elevated from the posterior tonsillar pillars on either side. Not shown: The uvula may be retracted for improved visualization. (B) Flaps are transposed into a horizontal direction to be inset into a transverse incision on the posterior pharyngeal wall. (C) The flaps are inset in an end-to-end fashion and the donor sites are sutured closed. The airway is smaller, but remains patent centrally. Note: For greater tightening of the sphincter, the flaps may be overlapped upon each other.

advocated elevation of more robust flaps, which included the palatopharyngeus muscle, bringing them together in an endto-end fashion. ${ }^{22}$ Several variations of this procedure have since been introduced, including notable techniques by Orticochea ${ }^{23}$ and Jackson. ${ }^{24}$ A modified version of Hynes' original technique remains one of the most popular variants of the sphincter pharyngoplasty utilized today.

Given the mobilization of the laterally based palatopharyngeus myomucosal flaps into the midline, the sphincter pharyngoplasty should be a favored procedure for correction of coronal or bowtie patterns of closure where lateral pharyngeal wall motion may be poor. Suggestions have also been made that this may be a more physiologic procedure than the pharyngeal flap, and that the sphincter itself may have some dynamic function due to its incorporation of muscle. However, these claims remain largely unproven.

The technique is performed with initial retraction of the uvula to obtain maximal visualization of the posterior pharynx, without division of the soft palate itself ( $\mathbf{- F i g . ~ 5 ) . ~ T h e ~}$ posterior tonsillar pillars (incorporating palatopharyngeus muscle and surrounding mucosa) are then incised and elevated superiorly. These superiorly-based flaps are raised as high as possible. A transverse incision is then made across the mucosa of the posterior pharynx, allowing a raw surface for inset of the flaps. The pharyngoplasty flaps can then be sutured end-to-end or overlapped significantly to further tighten the lateral walls and allow additional soft tissue bulk over the posterior pharyngeal wall. Following inset and suture of the flaps, the lateral donor sites are then closed directly.

\section{Furlow Palatoplasty}

The Furlow double-opposing Z-plasty repair of the palate was originally proposed as a means of primary cleft palate repair. ${ }^{25}$ Its elegant design had the additional benefit of addressing several issues related to subideal speech outcomes after cleft repair. Not only does it offer considerable palatal lengthening, ${ }^{26}$ it further corrects the abnormal anterior direction and insertion of the levator veli palatini muscles by repositioning the fibers into a transverse orientation. It is thought that lengthening the palate may allow it to more effectively span and occlude the velopharyngeal gap during speech production. Addressing the position of the muscle favors enhanced palatal mobility ${ }^{27}$ and has been shown to yield better velopharyngeal competence. ${ }^{28}$ Several studies have shown it to be efficacious as a secondary treatment for VPD resulting from a previously repaired cleft, ${ }^{29-31}$ or as a primary treatment for VPD due to a SMCP. ${ }^{32}$ When utilized for the correction of VPD, the Furlow technique has shown greatest success in the correction of smaller postoperative velopharyngeal gaps, ${ }^{33,34}$ which were estimated to be less than $1 \mathrm{~cm}$ in depth ${ }^{29}$ or demonstrating a small residual gap of $20 \%$ or less.

This technique has been compared with pharyngeal flap and sphincter pharyngoplasty, $6,35,36$ and there is evidence that the Furlow technique may offer superior outcomes in many situations. In general, the Furlow technique is preferred in palates that are kinetic, with evidence of anterior orientation of the levator muscle fibers. It offers a lower risk of obstructive sleep apnea than either the pharyngeal flap or sphincter pharyngoplasty, and has a low rate of oronasal fistulas. This is now the preferred first-line intervention at many institutions, though individual practice varies and evidence for a comprehensive treatment algorithm continues to accumulate. ${ }^{37}$

The secondary Furlow palatoplasty (-Fig. $\mathbf{6}$ ) is initially performed with identification of the hamuli prior to the injection of local anesthetic. The soft palate is then divided at the midline, typically along a previous scar from initial cleft repair, up to the region of the hard/soft palate junction. Oral Z-plasty incisions are then designed from the hamuli, with the posteriorly-based musculomucosal flap drawn to the posterior edge of the hard palate and the anteriorly-based mucosal flap extending posteriorly toward the divided uvula. The levator muscle is then carefully released from the posterior edge of the hard palate and separated from the nasal mucosa. During this process, the tensor veli palatini attachments are automatically divided and separated from the 


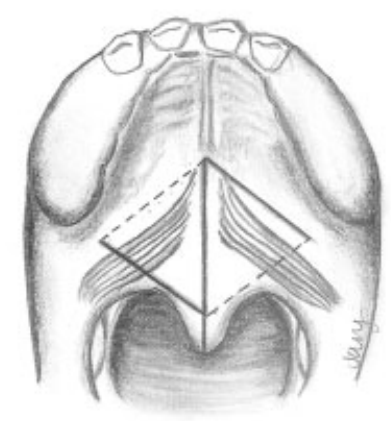

A

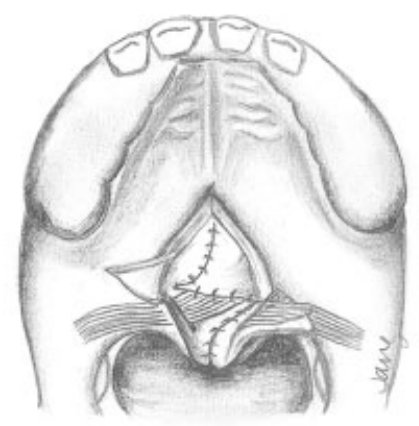

B

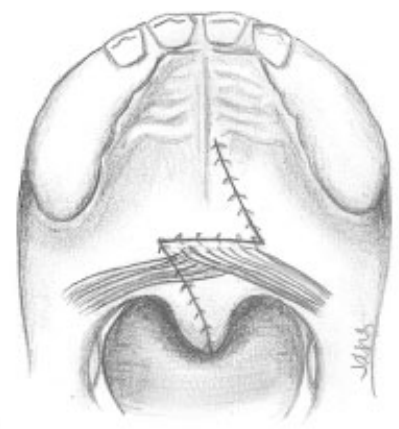

C

Fig. 6 Technique for secondary Furlow palatoplasty. (A) The palate is divided at the midline. Oral mucosal incisions (dark lines) and nasal mucosal incisions (dotted lines) are shown. On the right side, an oral musculomucosal flap will be elevated, whereas the left oral flap will contain mucosa only. (B) The nasal Z-plasty has been transposed and this layer has been closed. Note that the left side contains the nasal myomucosal flap, which is now transversely oriented. The oral mucosa flaps remain elevated. (C) The oral mucosa is now closed. The palate has been lengthened by the Z-plasties and the levator musculature has been transposed and overlapped upon itself.

levator. After myomucosal flap elevation, the nasal mucosal flap is then elevated on the ipsilateral side. This limb is incised from the base of the uvula to the lateral edge of the exposed levator.

Attention is turned to the opposite side, where an oral mucosal flap is elevated from the base of the uvula to the hamulus, using care to avoid any elevation of muscle. Following this, the final nasal myomucosal flap is developed. The muscle is carefully released from the hard palate and the nasal mucosa is divided, taking care to leave a small cuff of mucosa along the hard palate edge to suture to during closure. Once the dissection has been completed, the nasal Z-plasty flaps are transposed and sutured. The oral flaps are similarly transposed. In the process, the levator musculature is mobilized from an oblique orientation to a transverse dimension, with significant overlap of the muscle on the oral and nasal layers occurring.

\section{Palatal Re-Repair}

The concept of palatal re-repair has largely been advocated by Sommerlad $^{38,39}$ for the secondary correction of VPD in patients who demonstrated anterior insertion of the levator veli palatini. Utilizing an aggressive intravelar veloplasty approach whereby the velar musculature is radically dissected and retropositioned, the re-repair procedure has been successful in avoiding additional surgical intervention in $80 \%$ of cases. This provides an attractive alternative to the Furlow palatoplasty technique and argues for the importance of correction of the abnormal position of the levator. However, the idea of re-repair has garnered less popularity than the Furlow procedure and little confirmatory data are yet available from other institutions documenting similar results.

\section{Posterior Pharyngeal Wall Augmentation}

Correction of VPD by augmentation of the posterior pharyngeal wall has been attempted intermittently since the late 1800 s. Conceptually, augmentation of the posterior pharyngeal wall should bring this structure closer to the velum during maximal closure of the velum, thereby aiding in speech, especially for smaller velopharyngeal defects. Passavant ${ }^{40}$ described an unsuccessful attempt to do so utilizing adjacent soft tissues in 1879. Since then, a myriad of other products have been tried in an attempt to optimize speech function. This has included petroleum jelly, ${ }^{41}$ paraffin, ${ }^{42}$ cartilage, ${ }^{43-45}$ fat and/or fascia, ${ }^{46,47}$ silastic, ${ }^{48,49}$ Teflon, ${ }^{50}$ and Proplast. ${ }^{51}$ Numerous complications have been documented with such procedures, including infection, exposure, extrusion, migration, and embolism. The results have remained largely unimpressive and the procedure has yet to be accepted as a mainstay of treatment.

\section{Acknowledgments}

The author would like to acknowledge the work of Dr. Judy L. Jang who created the illustrations for this article.

\section{References}

1 Lewis JR, Andreassen ML, Leeper HA, Macrae DL, Thomas J. Vocal characteristics of children with cleft lip/palate and associated velopharyngeal incompetence. J Otolaryngol 1993;22(2):113-117

2 Paal S, Reulbach U, Strobel-Schwarthoff K, Nkenke E, Schuster M. Evaluation of speech disorders in children with cleft lip and palate. J Orofac Orthop 2005;66(4):270-278

3 Trost-Cardamone JE. Coming to terms with VPI: a response to Loney and Bloem. Cleft Palate J 1989;26(1):68-70

4 Witt PD, Wahlen JC, Marsh JL, Grames LM, Pilgram TK. The effect of surgeon experience on velopharyngeal functional outcome following palatoplasty: is there a learning curve? Plast Reconstr Surg 1998;102(5):1375-1384

5 Sell D, Grunwell P, Mildinhall S, et al. Cleft lip and palate care in the United Kingdom-the Clinical Standards Advisory Group (CSAG) Study. Part 3: speech outcomes. Cleft Palate Craniofac J 2001;38 (1):30-37

6 Sullivan SR, Vasudavan S, Marrinan EM, Mulliken JB. Submucous cleft palate and velopharyngeal insufficiency: comparison of speech outcomes using three operative techniques by one surgeon. Cleft Palate Craniofac J 2011;48(5):561-570

7 Havstam C, Lohmander A, Persson C, Dotevall H, Lith A, Lilja J. Evaluation of VPI-assessment with videofluoroscopy and nasoendoscopy. Br J Plast Surg 2005;58(7):922-931

8 Sommerlad BC. Evaluation of VPI-assessment with videofluoroscopy and nasoendoscopy. Br J Plast Surg 2005;58(7):932-933 
9 Lipira AB, Grames LM, Molter D, Govier DP, Kane AA, Woo AS. Videofluoroscopic and nasendoscopic correlates of speech in velopharyngeal dysfunction. Cleft Palate Craniofac J 2011;48 (5):550-560

10 Golding-Kushner KJ, Argamaso RV, Cotton RT, et al. Standardization for the reporting of nasopharyngoscopy and multiview videofluoroscopy: a report from an International Working Group. Cleft Palate J 1990;27(4):337-347, discussion 347-348

11 Argamaso RV, Shprintzen RJ, Strauch B, et al. The role of lateral pharyngeal wall movement in pharyngeal flap surgery. Plast Reconstr Surg 1980;66(2):214-219

12 Marsh JL. The evaluation and management of velopharyngeal dysfunction. Clin Plast Surg 2004;31(2):261-269

13 Gosain AK, Arneja JS. Management of the black hole in velopharyngeal incompetence: combined use of a Furlow palatoplasty and sphincter pharyngoplasty. Plast Reconstr Surg 2007;119(5): 1538-1545

14 Ysunza A, Pamplona C, Ramírez E, Molina F, Mendoza M, Silva A. Velopharyngeal surgery: a prospective randomized study of pharyngeal flaps and sphincter pharyngoplasties. Plast Reconstr Surg 2002;110(6):1401-1407

15 Abyholm F, D'Antonio L, Davidson Ward SL, et al; VPI Surgical Group. Pharyngeal flap and sphincterplasty for velopharyngeal insufficiency have equal outcome at 1 . year postoperatively: results of a randomized trial. Cleft Palate Craniofac J 2005;42(5):501-511

16 Schoenborn K. Über eine neue Methode der Staphylorrhaphie. Verh Dtsch Ges Chir 1875;4:235-239

17 Schoenborn K. Vorstellung eines Falle von Staphyloplastik. Verh Dtsch Ges Chir 1886;15:57-62

18 Padgett EC. The repair of cleft palates after unsuccessful operations, with special reference to cases with an extensive loss of palatal tissue. Arch Surg 1930;20:453-472

19 Hogan VM. A clarification of the surgical goals in cleft palate speech and the introduction of the lateral port control (l.p.c.) pharyngeal flap. Cleft Palate J 1973;10:331-345

20 Shprintzen RJ, Lewin ML, Croft CB, et al. A comprehensive study of pharyngeal flap surgery: tailor made flaps. Cleft Palate J 1979;16 (1):46-55

21 Hynes W. Pharyngoplasty by muscle transplantation. Br J Plast Surg 1950;3(2):128-135

22 Hynes $\mathrm{W}$. The results of pharyngoplasty by muscle transplantation in failed cleft palate cases, with special reference to the influence of the pharynx on voice production; Hunterian lecture, 1953. Ann R Coll Surg Engl 1953;13(1):17-35

23 Orticochea M. Construction of a dynamic muscle sphincter in cleft palates. Plast Reconstr Surg 1968;41(4):323-327

24 Jackson IT, Silverton JS. The sphincter pharyngoplasty as a secondary procedure in cleft palates. Plast Reconstr Surg 1977;59 (4):518-524

25 Furlow LT Jr. Cleft palate repair by double opposing Z-plasty. Plast Reconstr Surg 1986;78(6):724-738

26 Guneren E, Uysal OA. The quantitative evaluation of palatal elongation after Furlow palatoplasty. J Oral Maxillofac Surg 2004;62(4):446-450

27 Honjo I, Harada H, Okazaki N. Significance of levator muscle sling formation in cleft palate surgery: evaluation by electrical stimulation. Plast Reconstr Surg 1980;65(4):443-446

28 Hassan ME, Askar S. Does palatal muscle reconstruction affect the functional outcome of cleft palate surgery? Plast Reconstr Surg 2007;119(6):1859-1865

29 Chen PK, Wu JT, Chen YR, Noordhoff MS. Correction of secondary velopharyngeal insufficiency in cleft palate patients with the Furlow palatoplasty. Plast Reconstr Surg 1994;94(7):933-941, discussion 942-943

30 Perkins JA, Lewis CW, Gruss JS, Eblen LE, Sie KC. Furlow palatoplasty for management of velopharyngeal insufficiency: a pro- spective study of 148 consecutive patients. Plast Reconstr Surg 2005;116(1):72-80, discussion 81-84

31 Sie KC, Tampakopoulou DA, Sorom J, Gruss JS, Eblen LE. Results with Furlow palatoplasty in management of velopharyngeal insufficiency. Plast Reconstr Surg 2001;108(1):17-25, discussion 26-29

32 Chen PK, Wu J, Hung KF, Chen YR, Noordhoff MS. Surgical correction of submucous cleft palate with Furlow palatoplasty. Plast Reconstr Surg 1996;97(6):1136-1146, discussion 1147-1149

33 D'Antonio LL, Eichenberg BJ, Zimmerman GJ, et al. Radiographic and aerodynamic measures of velopharyngeal anatomy and function following Furlow Z-plasty. Plast Reconstr Surg 2000;106 (3):539-549, discussion 550-553

34 Deren O, Ayhan M, Tuncel A, et al. The correction of velopharyngeal insufficiency by Furlow palatoplasty in patients older than 3 years undergoing Veau-Wardill-Kilner palatoplasty: a prospective clinical study. Plast Reconstr Surg 2005;116(1):85-93, discussion 94-96

35 Wójcicki P, Wójcicka K. Prospective evaluation of the outcome of velopharyngeal insufficiency therapy after pharyngeal flap, a sphincter pharyngoplasty, a double Z-plasty and simultaneous Orticochea and Furlow operations. J Plast Reconstr Aesthet Surg 2011;64(4):459-461

36 Dailey SA, Karnell MP, Karnell LH, Canady JW. Comparison of resonance outcomes after pharyngeal flap and Furlow doubleopposing z-plasty for surgical management of velopharyngeal incompetence. Cleft Palate Craniofac J 2006;43(1):38-43

37 Rottgers SA, Ford M, Cray J, et al. An algorithm for application of Furlow palatoplasty to the treatment of velocardiofacial syndrome-associated velopharyngeal insufficiency. Ann Plast Surg 2011;66(5):479-484

38 Sommerlad BC, Henley M, Birch M, Harland K, Moiemen N, Boorman JG. Cleft palate re-repair-a clinical and radiographic study of 32 consecutive cases. Br J Plast Surg 1994;47(6):406-410

39 Sommerlad BC, Mehendale FV, Birch MJ, Sell D, Hattee C, Harland K. Palate re-repair revisited. Cleft Palate Craniofac J 2002;39(3): 295-307

40 Passavant G. Über die Verbesserung der Sprache nach der Uranoplastik. Deutch Geselschaft Chirurgie 1879;23:771-780

41 Gersuny R. Über eine subcutane Prosthese. Zeitschrift fur Heilkunde 1900;21:199-204

42 Eckstein H. Hartparaffininjecktionen in der hintere Rachenwand bei angeborenen und etwarbenen Gaumendefekten. Deutsch med. Wochenschrift 1922;1:1186-1187

43 Hollweg E, Perthes G. Beitrag zur Behandlung von Gaumenspalten. Tübingen: Pietzcker, 1912

44 Bentley FH, Watkins II. Speech after repair of cleft palate. Lancet 1947;2(6485):862-865

45 Denny AD, Marks SM, Oliff-Carneol S. Correction of velopharyngeal insufficiency by pharyngeal augmentation using autologous cartilage: a preliminary report. Cleft Palate Craniofac J 1993;30:46-54

46 von Gaza WV. Transplanting of free fatty tissue in the retropharyngeal area in cases of cleft palate. Paper presented at: German Surgical Society, April 9, 1926; Berlin, Germany

47 Halle H. Gaumennaht und gaumenplastik. Ztschr Hals, Nasen-U. Ohrenheilk 1925;12:377-389

48 Blocksma R. Correction of velopharyngeal insufficiency by Silastic pharyngeal implant. Plast Reconstr Surg 1963;31:268-274

49 Blocksma R, Braley S. The silicones in plastic surgery. Plast Reconstr Surg 1965;35:366-370

50 Lewy R, Cole R, Wepman J. Teflon injection in the correction of velopharyngeal insufficiency. Ann Otol Rhinol Laryngol 1965;74 (3):874-879

51 Wolford LM, Oelschlaeger M, Deal R. Proplast as a pharyngeal wall implant to correct velopharyngeal insufficiency. Cleft Palate J 1989;26(2):119-126, discussion 126-128 